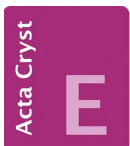

CRYSTALLOGRAPHIC COMMUNICATIONS

ISSN 2056-9890

\section{Crystal structure of poly $\left[\left\{\mu_{3}-(E)-3-[3-(\right.\right.$ carboxyl- atomethoxy)phenyl]acrylato- $\left.\kappa^{3} O, O^{\prime}: O^{\prime \prime}: O^{\prime \prime \prime}\right\}\left[\mu_{2}-3-\right.$ (pyridin-4-yl)-1H-pyrazole- $\left.\kappa^{2} N: N^{\prime}\right]$ cobalt(II)]}

\author{
Can Zhao, Xiao-Zong Li, Hong-Lan Kang and Yi-Hang Wen*
}

Zhejiang Key Laboratory for Reactive Chemistry on Solid Surfaces, Institute of Physical Chemistry, Zhejiang Normal University, Jinhua, Zhejiang 321004, People's Republic of China. *Correspondence e-mail: wyh@zjnu.edu.cn

Received 14 August 2016

Accepted 2 September 2016

Edited by S. Parkin, University of Kentucky, USA

The title compound, $\left[\mathrm{Co}\left(\mathrm{C}_{11} \mathrm{H}_{8} \mathrm{O}_{5}\right)\left(\mathrm{C}_{8} \mathrm{H}_{7} \mathrm{~N}_{3}\right)\right]_{n}$, which is based on (E)-3-[3(carboxymethoxy)phenyl]acrylic acid $\left(\mathrm{H}_{2} L\right)$ and 3-(pyridin-4-yl)pyrazole (pp) ligands, has been synthesized under solvothermal conditions. The dihedral angle between pyrazole and pyridine rings in the pp ligands is $23.1(2)^{\circ}$. In the crystal, helical chains formed by $\mathrm{pp}$ and $L$ ligands connected to the $\mathrm{Co}^{\mathrm{II}}$ atom propagate parallel to the $c$ axis. $\mathrm{Co}^{\mathrm{II}}$ atoms of adjacent chains are bridged by the acrylic acid groups of $L$ ligands into corrugated polymeric sheets in the ac plane.

\section{Chemical context}

The rational design and synthesis of metal-organic frameworks (MOFs) with multi-carboxylate ligands and metal atoms has attracted much attention in coordination chemistry due to the varied topologies and potential applications in catalysis, gas adsorption, photochemistry etc (Fernández et al., 2016). The versatility of metal-organic chemistry offers the opportunity to construct multifunctional materials based on the assembly of molecular building blocks. Much attention has been devoted to the cogitative design and control of selfassembly of infinite coordination networks by careful selection of ligand geometry (Liu et al., 2016; Yoon et al., 2012). In this regard, the use of symmetrical ligands has been a successful paradigm because of their structural predictability (Rosi et al., 2003; Luo et al., 2003). Incorporation of unsymmetrical ligands in such systems, however, is relatively recent (Wang et al., 2004; Chen et al., 2003; Qin et al., 2005). Compared to symmetrical ligands, ligands with two or more coordination sites with differing donor ability can lead to unsymmetrical ligands being assembled around metal atoms in diverse arrangements. This can result in unprecedented structures with novel topological features, such as a clay-like double layer (Pan et al., 2000), large spherical cavities and functional 1D channels (Shin et al., 2003). Although important progress has been made in the construction of coordination polymers by applying a single type of organic ligand, research involving a combination of more than one ligand is an especially attractive target, as it allows the construction of an almost infinite number of frameworks with different crystal structures.

In our work, we use (E)-3-[3-(carboxymethoxy)phenyl]acrylic acid $\left(\mathrm{H}_{2} L\right)$ and 3-(pyridin-4-yl)pyrazole (pp) as ligands to construct novel MOFs that are based on the following considerations: (1) the carboxylate group is conjugated with 
the benzene ring through a $\mathrm{C}=\mathrm{C}$ double bond, which makes the electron density delocalized in the ligand so that it may become more rigid when coordinating to metal ions, and have more coordination modes and conformation changes (Kong et al., 2013; Liu et al., 2010); (2) the presence of a phenolic hydroxyl group and benzene ring in the ligand allows the possibility of hydrogen bonding and $\pi-\pi$ stacking interactions in the crystal lattices; (3) the $\mathrm{N}$-donor ligand could enhance structural stability.

We herein report the synthesis and crystal structure of $\left[\mathrm{Co}\left(\mathrm{C}_{11} \mathrm{H}_{8} \mathrm{O}_{5}\right)\left(\mathrm{C}_{8} \mathrm{H}_{7} \mathrm{~N}_{3}\right)\right]_{n}$ based on these two mixed ligands.

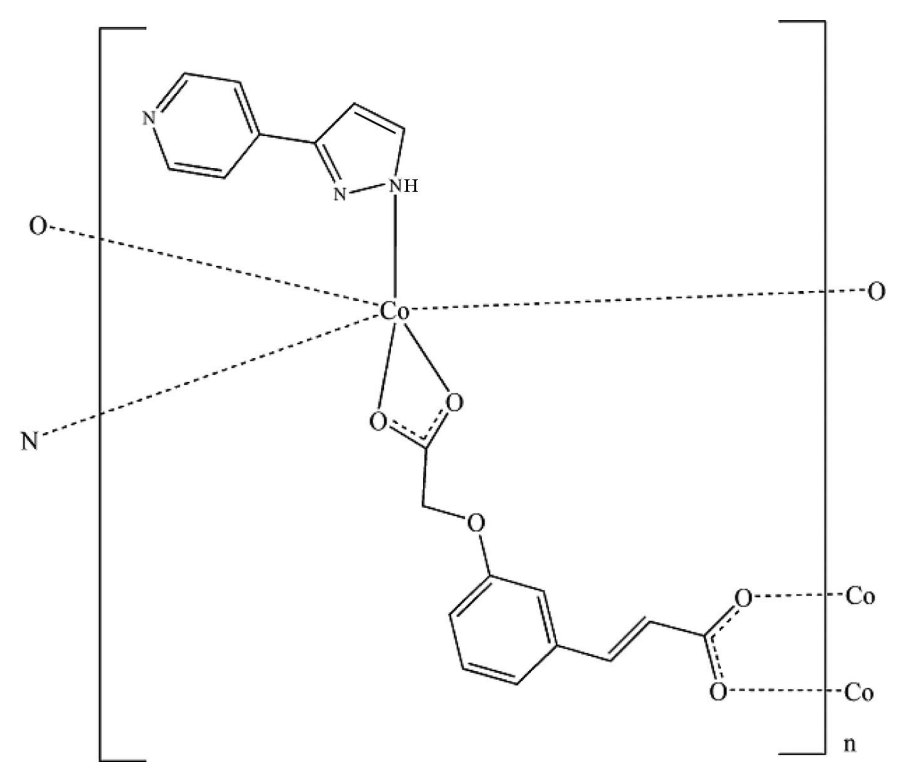

\section{Structural commentary}

As shown in Fig. 1, the asymmetric unit of the title compound comprises one $\mathrm{Co}^{2+}$ cation, one fully deprotonated $L^{2-}$ anion,

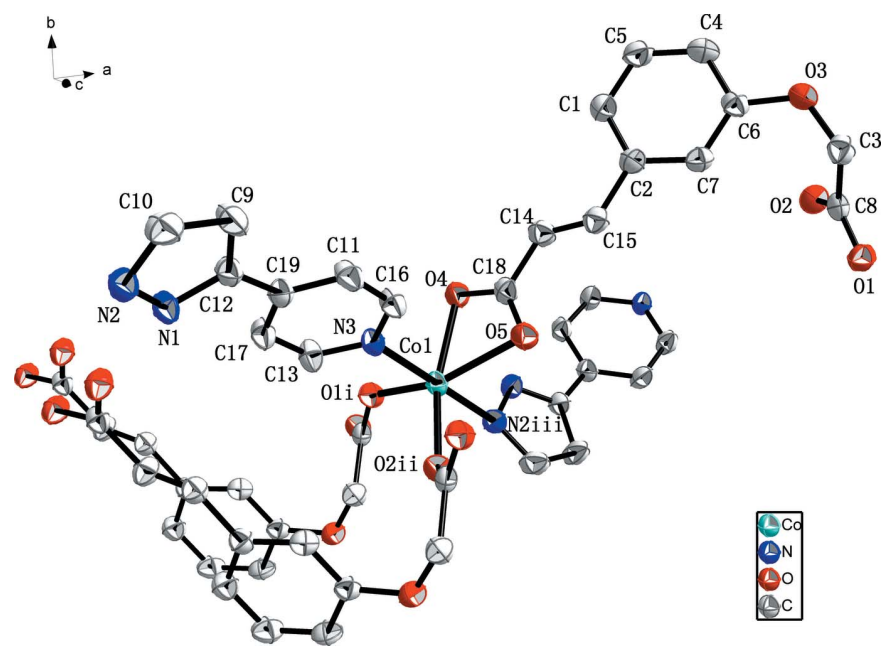

Figure 1

The coordination environment of the $\mathrm{Co}^{2+}$ ion in the title complex (omitting all $\mathrm{H}$ atoms), showing the atom-numbering scheme for non- $\mathrm{H}$ atoms. Displacement ellipsoids are drawn at the $40 \%$ probability level. [Symmetry codes: (i) $x, y, z-1$; (ii) $x-\frac{1}{4}, \frac{1}{4}-y, z+\frac{3}{4}$; (iii) $x+\frac{1}{4}, \frac{1}{4}-y, z+\frac{1}{4}$ ] and one pp ligand. The $\mathrm{Co}^{\mathrm{II}}$ atom has a distorted octahedral geometry, coordinated by four $\mathrm{O}$ atoms from three $L^{2-}$ ligands, with $\mathrm{Co}^{\mathrm{II}}-\mathrm{O}$ distances of 2.037 (2)-2.252 (2) $\AA$, and two $\mathrm{N}$ atoms from two pp ligands with $\mathrm{Co}^{\mathrm{II}}-\mathrm{N}$ distances of 2.130 (2) and 2.158 (3) $\AA$. The $L^{2-}$ ligand adopts two different coordination modes. In this structure, the dihedral angles between the rings in the pp ligands is $23.1(2)^{\circ}$. The $1 \mathrm{D}$ helical chains (Fig. 2) are assembled by $\mathrm{Co}^{2+}$ cations, pp ligands and $L$ ligands. Helical chains along the $c$ axis are connected to adjacent chains by $L$ ligands that bridge the $\mathrm{Co}^{\mathrm{II}}$ atoms, forming a two-dimensional polymeric structure in the $a c$ plane (Fig. 3).

In the structure, every $\eta^{3}-(E)-3-[3-($ carboxymethoxy)phenyl]acrylic acid ligand is connected to three Co atoms, while every $\eta^{3}$-3-(pyridin-4-yl)pyrazole is connected to two Co atoms. The $\mathrm{Co}^{\mathrm{II}}$ atom connects three $L^{2-}$ ligands and two pp ligands, and so can be described as a five-connected node. Thus, the topology of the structure could be given simply as a $(2,3,5)$-connected network.

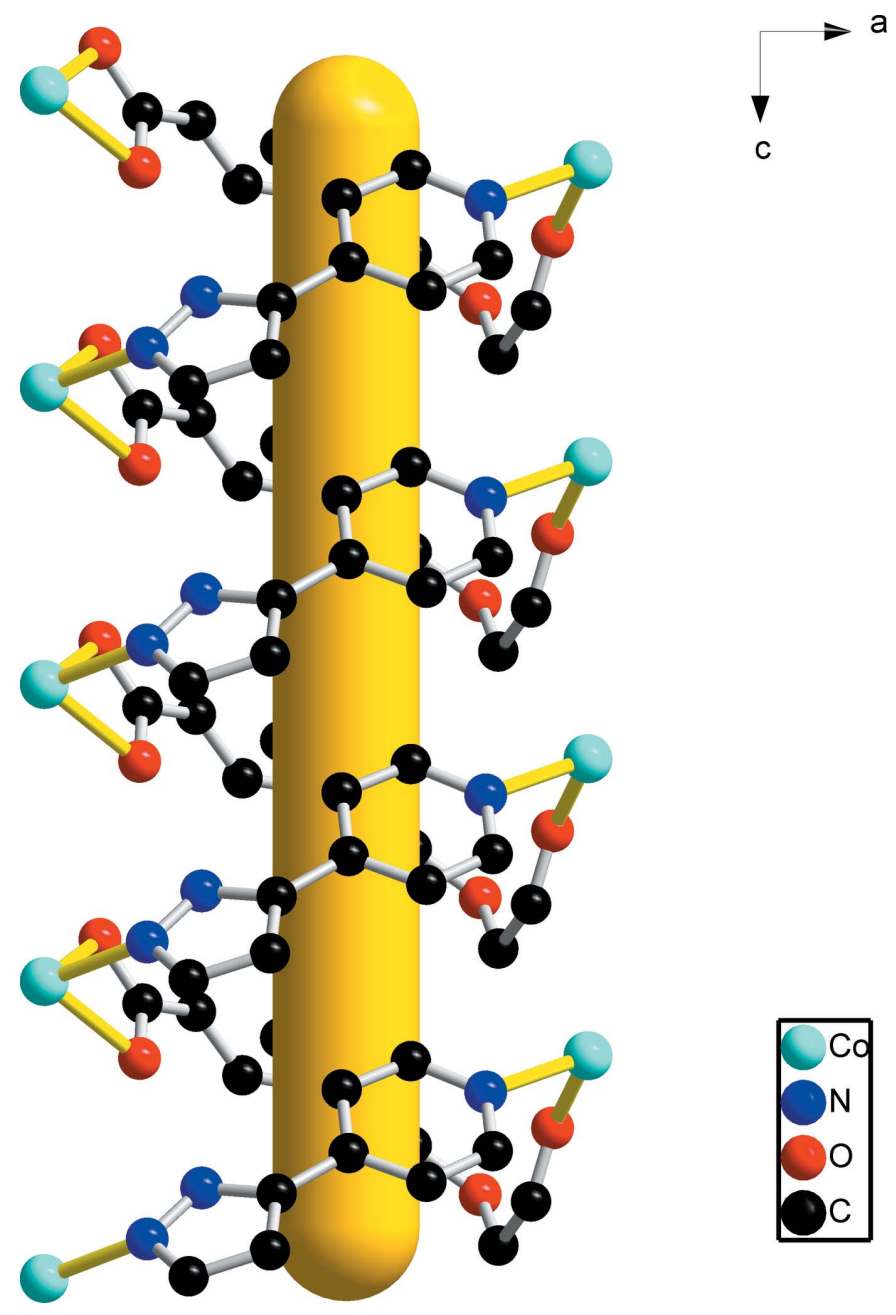

Figure 2

The helical chain in the title compound (omitting all $\mathrm{H}$ atoms). The yellow rod indicates the direction of propagation of the helix (i.e. parallel to the $c$ axis). 
Table 1

Hydrogen-bond geometry $\left(\AA,^{\circ}\right)$.

\begin{tabular}{lllll}
\hline$D-\mathrm{H} \cdots A$ & $D-\mathrm{H}$ & $\mathrm{H} \cdots A$ & $D \cdots A$ & $D-\mathrm{H} \cdots A$ \\
\hline $\mathrm{N} 1-\mathrm{H} 1 A \cdots \mathrm{O}^{\mathrm{i}}$ & 0.86 & 2.05 & $2.869(3)$ & 159 \\
\hline
\end{tabular}

Symmetry code: (i) $x-\frac{1}{4},-y+\frac{1}{4}, z-\frac{1}{4}$

\section{Supramolecular features}

In this structure, $L$ ligands form hydrogen bonds to the $\mathrm{pp}$ ligands, thereby enhancing the polymer stability (Table 1 and Fig. 3). The polymer interactions consist of N1(pyrazole) H1A ‥ O5 $\left(x-\frac{1}{4},-y+\frac{1}{4}, z-\frac{1}{4}\right)$ hydrogen bonds where each $L$ ligand makes a hydrogen bond with a neighboring pp ligand.

\section{Database survey}

The crystal structure of a 2D polymeric Cd-containing compound with (E)-3-(3-carboxymethoxy)phenyl)acrylic acid and 1,3-di-pyridin-4-ylpropane ligands (the Cd-crystal), recently reported by Wang et al. (2014), has a similar structure to the title compound. Both structures include hydrogen bonds, though in the Cd-crystal, these are $\mathrm{O}-\mathrm{H} \cdots \mathrm{O}$ hydrogen bonds rather than $\mathrm{N}-\mathrm{H} \cdots \mathrm{O}$ as in the title compound.

\section{Synthesis and crystallization}

All of the chemical reagents and solvents are commercially available and used without further purification. Elemental analyses were carried out on a Perkin-Elmer 2400 Series II analyzer.

\section{Synthesis of $\left[\mathrm{Co}\left(\mathrm{C}_{11} \mathrm{H}_{8} \mathrm{O}_{5}\right)\left(\mathrm{C}_{8} \mathrm{H}_{7} \mathrm{~N}_{3}\right)\right]_{n}$}

(1): A mixture of $\mathrm{CoCl}_{2} \cdot 6 \mathrm{H}_{2} \mathrm{O}(0.1185 \mathrm{~g}, 0.5 \mathrm{mmol}), \mathrm{H}_{2} L$ (Zheng et al., 2011; Fu \& Wen, 2011) (0.222 g, $1 \mathrm{mmol})$ and pp
Table 2

Experimental details.

\begin{tabular}{|c|c|}
\hline \multicolumn{2}{|l|}{ Crystal data } \\
\hline Chemical formula & {$\left[\mathrm{Co}\left(\mathrm{C}_{11} \mathrm{H}_{8} \mathrm{O}_{5}\right)\left(\mathrm{C}_{8} \mathrm{H}_{7} \mathrm{~N}_{3}\right)\right]$} \\
\hline$M_{\mathrm{r}}$ & 424.27 \\
\hline Crystal system, space group & Orthorhombic, $F d d 2$ \\
\hline Temperature $(\mathrm{K})$ & 296 \\
\hline$a, b, c(\AA)$ & $\begin{array}{l}35.4631(11), 40.2873(12) \\
\quad 4.8423(1)\end{array}$ \\
\hline$V\left(\AA^{3}\right)$ & $6918.3(3)$ \\
\hline$Z$ & 16 \\
\hline Radiation type & Мо $K \alpha$ \\
\hline$\mu\left(\mathrm{mm}^{-1}\right)$ & 1.03 \\
\hline Crystal size $(\mathrm{mm})$ & $0.24 \times 0.12 \times 0.06$ \\
\hline \multicolumn{2}{|l|}{ Data collection } \\
\hline Diffractometer & Bruker APEXII CCD \\
\hline Absorption correction & $\begin{array}{l}\text { Multi-scan (SADABS; Bruker, } \\
\text { 2014) }\end{array}$ \\
\hline$T_{\min }, T_{\max }$ & $0.861,0.943$ \\
\hline $\begin{array}{l}\text { No. of measured, independent and } \\
\text { observed }[I>2 \sigma(I)] \text { reflections }\end{array}$ & $15248,3915,3425$ \\
\hline$R_{\text {int }}$ & 0.036 \\
\hline$(\sin \theta / \lambda)_{\max }\left(\AA^{-1}\right)$ & 0.651 \\
\hline \multicolumn{2}{|l|}{ Refinement } \\
\hline$R\left[F^{2}>2 \sigma\left(F^{2}\right)\right], w R\left(F^{2}\right), S$ & $0.029,0.061,1.02$ \\
\hline No. of reflections & 3915 \\
\hline No. of parameters & 253 \\
\hline No. of restraints & 1 \\
\hline $\mathrm{H}$-atom treatment & $\mathrm{H}$-atom parameters constrained \\
\hline$\Delta \rho_{\max }, \Delta \rho_{\min }\left(\mathrm{e} \AA^{-3}\right)$ & $0.18,-0.24$ \\
\hline Absolute structure & $\begin{array}{l}\text { Flack } x \text { determined using } 1316 \\
\quad \text { quotients }\left[\left(I^{+}\right)-\left(I^{-}\right)\right] /\left[\left(I^{+}\right)+\left(I^{-}\right)\right] \\
\quad \text { (Parsons } \text { et al., } 2013)\end{array}$ \\
\hline Absolute structure parameter & $0.025(8)$ \\
\hline
\end{tabular}

Computer programs: APEX2 and SAINT-Plus (Bruker, 2014), SHELXS97 and SHELXTL (Sheldrick, 2008), SHELXL2014 (Sheldrick, 2015) and publCIF (Westrip, 2010).

(0.1451 g, $1 \mathrm{mmol})$ were dissolved in $22 \mathrm{~mL} \mathrm{H}_{2} \mathrm{O} / \mathrm{CH}_{3} \mathrm{OH}(v / v$, 10:1) mixed solvent. The $\mathrm{pH}$ value was adjusted to 7 by adding to a few drops of an aqueous $\mathrm{NaOH}$ solution $\left(2.0 \mathrm{~mol} \mathrm{~L}^{-1}\right)$. It

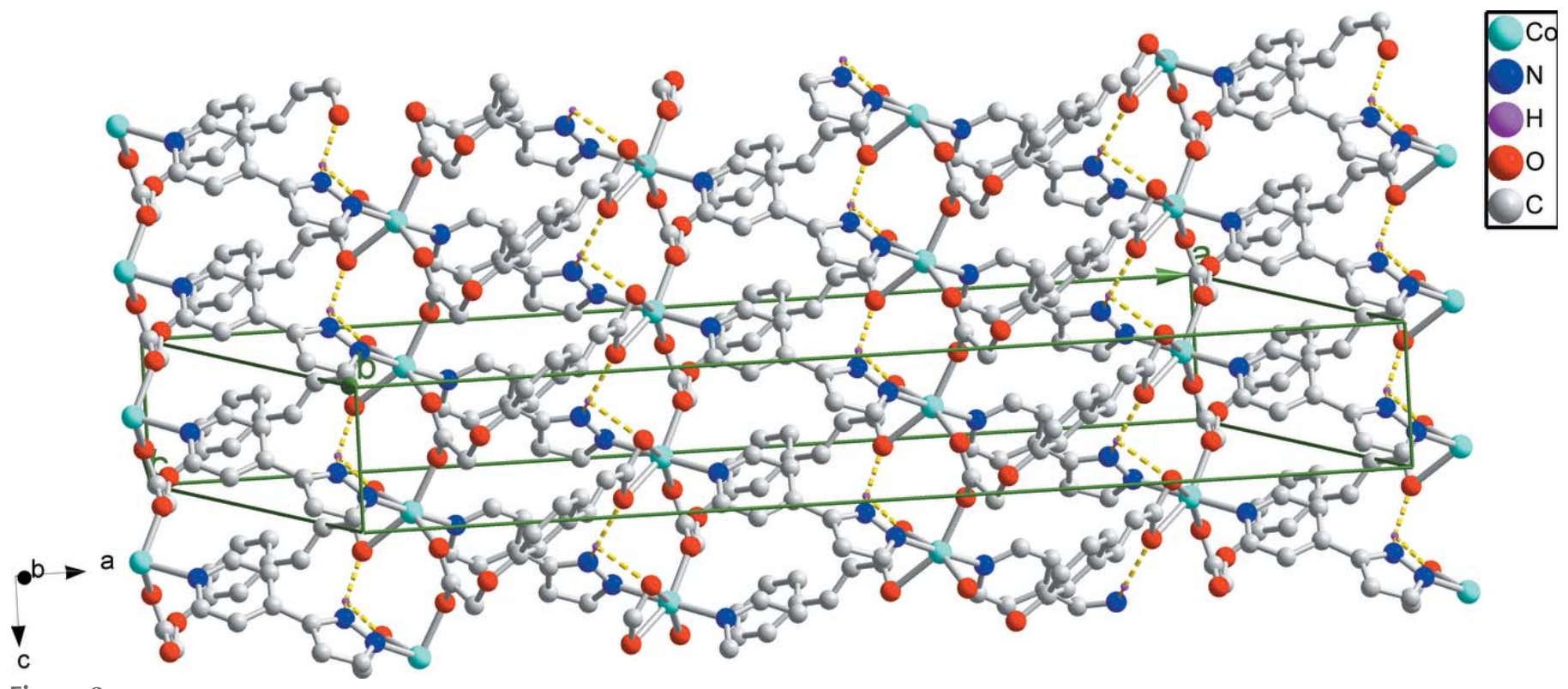

Figure 3

The two-dimensional packing of the title compound. Hydrogen bonds are depicted as dashed lines. 
was then sealed in a $25 \mathrm{~mL}$ stainless steel reactor and heated to $433 \mathrm{~K}$ for three days. The mixture was then cooled to room temperature at a rate of $5 \mathrm{~K} \mathrm{~h}^{-1}$, and red block-shaped crystals were obtained (yield: $62 \%$ based on $\mathrm{Co}$ ). Analysis calculated (\%) for $\mathrm{C}_{19} \mathrm{H}_{15} \mathrm{CoN}_{3} \mathrm{O}_{5}$ (424.27): $\mathrm{C} 53.81, \mathrm{H} 3.62, \mathrm{~N}$ 9.85; found (\%): C 53.79, H 3.56, N 9.90. IR data $\left(\mathrm{KBr}, \mathrm{cm}^{-1}\right)$ : 3432, 1649, 1501, 1407, 1274, 1206, 1180, 1086, 978, 844, 724, 603.

\section{Refinement}

Crystal data, data collection and structure refinement details are summarized in Table 2. Hydrogen atoms attached to carbon atoms were refined using a riding-model approximation, with $U_{\text {iso }}(\mathrm{H})=1.2 U_{\text {eq }}(\mathrm{C})$ and $\mathrm{C}-\mathrm{H}=0.93 \AA$ (aromatic and carbene) and $0.97 \AA$ (methylene). Other hydrogen atoms were located in difference electron-density maps and refined freely.

\section{Acknowledgements}

This work was supported financially by the Open Research Fund of Top Key Discipline of Chemistry in Zhejiang Provincial Colleges and the Key Laboratory of the Ministry of Education for Advanced Catalysis Materials (ZJHX201515).

\section{References}

Bruker (2014). APEX2, SAINT and SADABS. Bruker AXS Inc., Madison, Wisconsin, USA.
Chen, W., Yue, Q., Chen, C., Yuan, H. M., Xu, W., Chen, J. S. \& Wang, S. N. (2003). Dalton Trans. pp. 28-30.

Fernández, B., Beobide, G., Sánchez, I., Carrasco-Marín, F., Seco, J. M., Calahorro, A. J., Cepeda, J. \& Rodríguez-Diéguez, A. (2016). CrystEngComm, 18, 1282-1294.

Fu, J.-D. \& Wen, Y.-H. (2011). Acta Cryst. E67, o167.

Kong, G. Q., Han, Z. D., He, Y. B., Ou, S., Zhou, W., Yildirim, T., Krishna, R., Zou, C., Chen, B. \& Wu, C. D. (2013). Chem. Eur. J. 19, 14886-14894.

Liu, X. B., Lin, H., Xiao, Z. Y., Fan, W. D., Huang, A., Wang, R. M., Zhang, L. L. \& Sun, D. F. (2016). Dalton Trans. 45, 3743-3749.

Liu, D. M., Xie, Z. G., Ma, L. Q. \& Lin, W. B. (2010). Inorg. Chem. 49, 9107-9109.

Luo, J. H., Hong, M. C., Wang, R. H., Cao, R., Han, L., Yuan, D. Q., Lin, Z. Z. \& Zhou, Y. F. (2003). Inorg. Chem. 42, 4486-4488.

Pan, L., Huang, X. Y., Li, J., Wu, Y. G. \& Zheng, N. W. (2000). Angew. Chem. 112, 537-540.

Parsons, S., Flack, H. D. \& Wagner, T. (2013). Acta Cryst. B69, 249 259.

Qin, C., Wang, X. L., Li, Y. G., Wang, E. B., Su, Z. M., Xu, L. \& Clérac, R. (2005). Dalton Trans. pp. 2609-2614.

Rosi, N. L., Eckert, J., Eddaoudi, M., Vodak, M. T., Kim, J., O'Keeffe, M. \& Yaghi, O. M. (2003). Science, 300, 1127-1129.

Sheldrick, G. M. (2008). Acta Cryst. A64, 112-122.

Sheldrick, G. M. (2015). Acta Cryst. C71, 3-8.

Shin, D. M., Lee, I. S., Chung, Y. K. \& Lah, M. S. (2003). Inorg. Chem. 42, 5459-5461.

Wang, X. L., Qin, C., Wang, E. B., Li, Y. G., Hao, N., Hu, C. \& Xu, L. (2004). Inorg. Chem. 43, 1850-1856.

Wang, G. X., Zhang, Q. W. \& Wen, Y. H. (2014). Chinese J. Inorg. Chem. 30, 2571-2576.

Westrip, S. P. (2010). J. Appl. Cryst. 43, 920-925.

Yoon, M., Srirambalaji, R. \& Kim, K. (2012). Chem. Rev. 112, 11961231.

Zheng, X. Y., Ye, L. \& Wen, Y. H. (2011). J. Mol. Struct. 987, 132-137. 


\section{supporting information}

Acta Cryst. (2016). E72, 1425-1428 [https://doi.org/10.1107/S205698901601402X]

Crystal structure of poly[ $\left[\mu_{3}-(E)-3-[3-(\right.$ carboxylatomethoxy) phenyl] acrylato$\left.\kappa^{3} O, O^{\prime}: O^{\prime \prime}: O^{\prime \prime \prime}\right\}\left[\mu_{2}-3-\left(\right.\right.$ pyridin-4-yl)-1H-pyrazole- $\left.\kappa^{2} N: N^{\prime}\right]$ cobalt(II)]

\section{Can Zhao, Xiao-Zong Li, Hong-Lan Kang and Yi-Hang Wen}

Computing details

Data collection: APEX2 (Bruker, 2014); cell refinement: SAINT-Plus (Bruker, 2014); data reduction: SAINT-Plus (Bruker, 2014); program(s) used to solve structure: SHELXS97 (Sheldrick, 2008); program(s) used to refine structure:

SHELXL2014 (Sheldrick, 2015); molecular graphics: SHELXTL (Sheldrick, 2008); software used to prepare material for publication: publCIF (Westrip, 2010).

Poly $\left[\left\{\mu_{3}-(E)-3-\left[3-\left(\right.\right.\right.\right.$ carboxylatomethoxy) phenyl] acrylato- $\left.\kappa^{3} O, O^{\prime}: O^{\prime \prime}: O^{\prime \prime \prime}\right\}\left[\mu_{2}-3\right.$-(pyridin-4-yl)-1H-pyrazole$\left.\kappa^{2} N: N^{\prime}\right]$ cobalt(II)]

Crystal data

$\left[\mathrm{Co}\left(\mathrm{C}_{11} \mathrm{H}_{8} \mathrm{O}_{5}\right)\left(\mathrm{C}_{8} \mathrm{H}_{7} \mathrm{~N}_{3}\right)\right]$

$M_{r}=424.27$

Orthorhombic, $F d d 2$

$a=35.4631(11) \AA$

$b=40.2873(12) \AA$

$c=4.8423(1) \AA$

$V=6918.3(3) \AA^{3}$

$Z=16$

$F(000)=3472$

Data collection

Bruker APEXII CCD diffractometer

$\omega$ and $\varphi$ scans

Absorption correction: multi-scan

(SADABS; Bruker, 2014)

$T_{\text {min }}=0.861, T_{\max }=0.943$

15248 measured reflections

\section{Refinement}

Refinement on $F^{2}$

Least-squares matrix: full

$R\left[F^{2}>2 \sigma\left(F^{2}\right)\right]=0.029$

$w R\left(F^{2}\right)=0.061$

$S=1.02$

3915 reflections

253 parameters

1 restraint

Primary atom site location: structure-invariant direct methods
$D_{\mathrm{x}}=1.629 \mathrm{Mg} \mathrm{m}^{-3}$

Mo $K \alpha$ radiation, $\lambda=0.71073 \AA$

Cell parameters from 4631 reflections

$\theta=1.5-27.6^{\circ}$

$\mu=1.03 \mathrm{~mm}^{-1}$

$T=296 \mathrm{~K}$

Block, red

$0.24 \times 0.12 \times 0.06 \mathrm{~mm}$

3915 independent reflections

3425 reflections with $I>2 \sigma(I)$

$R_{\text {int }}=0.036$

$\theta_{\max }=27.6^{\circ}, \theta_{\min }=1.5^{\circ}$

$h=-46 \rightarrow 40$

$k=-48 \rightarrow 52$

$l=-6 \rightarrow 6$

Secondary atom site location: difference Fourier map

Hydrogen site location: inferred from neighbouring sites

$\mathrm{H}$-atom parameters constrained

$w=1 /\left[\sigma^{2}\left(F_{\mathrm{o}}^{2}\right)+(0.0282 P)^{2}\right]$

where $P=\left(F_{\mathrm{o}}{ }^{2}+2 F_{\mathrm{c}}{ }^{2}\right) / 3$

$(\Delta / \sigma)_{\max }=0.001$

$\Delta \rho_{\max }=0.18 \mathrm{e} \AA^{-3}$

$\Delta \rho_{\min }=-0.24$ e $\AA^{-3}$ 
Absolute structure: Flack $x$ determined using

1316 quotients $\left[\left(I^{+}\right)-\left(I^{-}\right)\right] /\left[\left(I^{+}\right)+\left(I^{-}\right)\right]$(Parsons et al., 2013)

Absolute structure parameter: $0.025(8)$

\section{Special details}

Geometry. All esds (except the esd in the dihedral angle between two 1.s. planes) are estimated using the full covariance matrix. The cell esds are taken into account individually in the estimation of esds in distances, angles and torsion angles; correlations between esds in cell parameters are only used when they are defined by crystal symmetry. An approximate (isotropic) treatment of cell esds is used for estimating esds involving l.s. planes.

Fractional atomic coordinates and isotropic or equivalent isotropic displacement parameters $\left(\AA^{2}\right)$

\begin{tabular}{|c|c|c|c|c|}
\hline & $x$ & $y$ & $z$ & $U_{\text {iso }} * / U_{\text {eq }}$ \\
\hline Co1 & $0.43282(2)$ & $0.10099(2)$ & $0.42130(8)$ & 0.02757 (11) \\
\hline N1 & $0.25519(6)$ & $0.16432(6)$ & $0.8665(5)$ & $0.0315(6)$ \\
\hline $\mathrm{H} 1 \mathrm{~A}$ & 0.2498 & 0.1481 & 0.7576 & $0.038^{*}$ \\
\hline $\mathrm{N} 2$ & $0.23011(7)$ & $0.17869(6)$ & $1.0382(6)$ & $0.0344(6)$ \\
\hline N3 & $0.38578(7)$ & $0.13040(6)$ & $0.5432(5)$ & $0.0302(6)$ \\
\hline $\mathrm{O} 1$ & $0.65789(6)$ & $0.15999(5)$ & $1.3036(4)$ & $0.0351(5)$ \\
\hline $\mathrm{O} 2$ & $0.66654(5)$ & $0.18826(5)$ & $0.9144(4)$ & $0.0334(5)$ \\
\hline $\mathrm{O} 3$ & $0.63324(6)$ & $0.24391(5)$ & $1.1390(5)$ & $0.0379(5)$ \\
\hline $\mathrm{O} 4$ & $0.45839(6)$ & $0.14604(5)$ & 0.2794 (4) & $0.0361(5)$ \\
\hline O5 & $0.47664(6)$ & $0.12681(5)$ & $0.6803(5)$ & $0.0359(5)$ \\
\hline $\mathrm{C} 1$ & $0.54127(9)$ & $0.24388(8)$ & $0.6113(6)$ & $0.0388(8)$ \\
\hline H1B & 0.5214 & 0.2445 & 0.4858 & $0.047^{*}$ \\
\hline $\mathrm{C} 2$ & $0.54793(8)$ & $0.21527(7)$ & $0.7657(6)$ & $0.0317(7)$ \\
\hline $\mathrm{C} 3$ & $0.64159(9)$ & $0.21627(7)$ & $1.3104(6)$ & $0.0353(7)$ \\
\hline $\mathrm{H} 3 \mathrm{~A}$ & 0.6188 & 0.2100 & 1.4085 & $0.042 *$ \\
\hline $\mathrm{H} 3 \mathrm{~B}$ & 0.6600 & 0.2231 & 1.4470 & $0.042 *$ \\
\hline $\mathrm{C} 4$ & $0.59389(9)$ & $0.27054(8)$ & $0.8261(7)$ & $0.0376(8)$ \\
\hline $\mathrm{H} 4 \mathrm{~A}$ & 0.6088 & 0.2893 & 0.8492 & $0.045^{*}$ \\
\hline $\mathrm{C} 5$ & $0.56410(9)$ & $0.27116(8)$ & $0.6447(8)$ & $0.0418(8)$ \\
\hline $\mathrm{H} 5 \mathrm{~A}$ & 0.5593 & 0.2903 & 0.5432 & $0.050^{*}$ \\
\hline $\mathrm{C} 6$ & $0.60175(8)$ & $0.24195(7)$ & $0.9746(6)$ & $0.0307(7)$ \\
\hline $\mathrm{C} 7$ & $0.57842(8)$ & $0.21459(7)$ & $0.9493(7)$ & $0.0319(7)$ \\
\hline $\mathrm{H} 7 \mathrm{~A}$ & 0.5830 & 0.1957 & 1.0545 & $0.038^{*}$ \\
\hline C8 & $0.65663(7)$ & $0.18596(7)$ & $1.1621(7)$ & $0.0280(6)$ \\
\hline $\mathrm{C} 9$ & $0.28655(9)$ & $0.20335(8)$ & $1.0763(8)$ & $0.0433(9)$ \\
\hline H9A & 0.3054 & 0.2178 & 1.1346 & $0.052 *$ \\
\hline $\mathrm{C} 10$ & $0.24921(9)$ & $0.20257(8)$ & $1.1639(8)$ & $0.0421(8)$ \\
\hline H10A & 0.2390 & 0.2170 & 1.2940 & $0.050 *$ \\
\hline C11 & $0.35849(9)$ & $0.17039(8)$ & $0.8458(7)$ & $0.0374(8)$ \\
\hline H11A & 0.3621 & 0.1865 & 0.9809 & $0.045^{*}$ \\
\hline C12 & $0.28963(8)$ & $0.17826(7)$ & $0.8854(7)$ & $0.0319(7)$ \\
\hline $\mathrm{C} 13$ & $0.35135(8)$ & $0.12506(8)$ & $0.4394(7)$ & $0.0362(7)$ \\
\hline H13A & 0.3488 & 0.1099 & 0.2961 & $0.043^{*}$ \\
\hline C14 & $0.50252(9)$ & $0.17858(8)$ & $0.5230(7)$ & $0.0370(7)$ \\
\hline $\mathrm{H} 14 \mathrm{~A}$ & 0.5028 & 0.1935 & 0.3763 & $0.044 *$ \\
\hline
\end{tabular}




$\begin{array}{lllll}\text { C15 } & 0.52392(8) & 0.18555(8) & 0.7372(6) & 0.0342(8) \\ \text { H15A } & 0.5239 & 0.1705 & 0.8827 & 0.041^{*} \\ \text { C16 } & 0.38898(9) & 0.15306(7) & 0.7424(6) & 0.0352(8) \\ \text { H16A } & 0.4128 & 0.1574 & 0.8150 & 0.042^{*} \\ \text { C17 } & 0.31952(9) & 0.14106(8) & 0.5349(7) & 0.0363(8) \\ \text { H17A } & 0.2961 & 0.1366 & 0.4568 & 0.044^{*} \\ \text { C18 } & 0.47794(8) & 0.14901(8) & 0.4955(6) & 0.0313(7) \\ \text { C19 } & 0.32276(8) & 0.16387(8) & 0.7491(6) & 0.0309(7)\end{array}$

Atomic displacement parameters $\left(\AA^{2}\right)$

\begin{tabular}{|c|c|c|c|c|c|c|}
\hline & $U^{11}$ & $U^{22}$ & $U^{33}$ & $U^{12}$ & $U^{13}$ & $U^{23}$ \\
\hline Col & 0.02385 (19) & $0.0318(2)$ & $0.02701(18)$ & $-0.00149(18)$ & $0.00226(17)$ & $0.00073(18)$ \\
\hline N1 & $0.0247(13)$ & $0.0366(15)$ & $0.0333(15)$ & $-0.0018(11)$ & $0.0050(10)$ & $-0.0072(11)$ \\
\hline $\mathrm{N} 2$ & $0.0285(14)$ & $0.0358(15)$ & $0.0387(14)$ & $0.0004(12)$ & $0.0066(12)$ & $-0.0056(12)$ \\
\hline N3 & $0.0253(14)$ & $0.0347(14)$ & $0.0306(12)$ & $-0.0022(12)$ & $0.0030(11)$ & $0.0001(11)$ \\
\hline $\mathrm{O} 1$ & $0.0423(13)$ & $0.0337(12)$ & $0.0295(10)$ & $0.0020(10)$ & $-0.0026(10)$ & $0.0017(10)$ \\
\hline $\mathrm{O} 2$ & $0.0352(11)$ & $0.0390(12)$ & $0.0260(10)$ & $0.0035(9)$ & $0.0074(10)$ & $-0.0010(10)$ \\
\hline $\mathrm{O} 3$ & $0.0402(12)$ & $0.0299(11)$ & $0.0436(13)$ & $-0.0026(9)$ & $-0.0066(11)$ & $-0.0044(10)$ \\
\hline $\mathrm{O} 4$ & $0.0354(13)$ & $0.0376(13)$ & $0.0353(11)$ & $-0.0051(10)$ & $-0.0053(10)$ & $0.0027(10)$ \\
\hline O5 & $0.0340(12)$ & $0.0394(12)$ & $0.0344(11)$ & $-0.0054(9)$ & $0.0011(10)$ & $0.0060(11)$ \\
\hline $\mathrm{C} 1$ & $0.0398(19)$ & 0.0368 (19) & $0.040(2)$ & $0.0030(15)$ & $-0.0050(14)$ & $0.0001(14)$ \\
\hline $\mathrm{C} 2$ & $0.0291(17)$ & $0.0310(17)$ & $0.0350(16)$ & $0.0008(14)$ & $0.0033(12)$ & $-0.0033(13)$ \\
\hline $\mathrm{C} 3$ & $0.0378(18)$ & $0.0386(19)$ & $0.0296(15)$ & $0.0037(15)$ & $-0.0030(14)$ & $-0.0058(14)$ \\
\hline $\mathrm{C} 4$ & $0.044(2)$ & 0.0277 (17) & 0.0409 (19) & $-0.0048(15)$ & $0.0034(15)$ & $-0.0002(13)$ \\
\hline $\mathrm{C} 5$ & $0.051(2)$ & $0.0308(17)$ & $0.0437(19)$ & $0.0012(15)$ & $-0.0009(17)$ & $0.0048(16)$ \\
\hline C6 & $0.0306(17)$ & $0.0311(16)$ & $0.0304(17)$ & $0.0005(14)$ & $0.0024(11)$ & $-0.0074(12)$ \\
\hline $\mathrm{C} 7$ & $0.0356(17)$ & $0.0272(15)$ & $0.0328(16)$ & $0.0013(12)$ & $0.0008(14)$ & $-0.0012(13)$ \\
\hline $\mathrm{C} 8$ & $0.0219(14)$ & $0.0351(16)$ & $0.0268(14)$ & $-0.0016(12)$ & $-0.0028(13)$ & $-0.0022(14)$ \\
\hline C9 & $0.0301(19)$ & 0.0379 (19) & $0.062(2)$ & $-0.0087(15)$ & $0.0071(16)$ & $-0.0138(16)$ \\
\hline $\mathrm{C} 10$ & $0.0426(19)$ & $0.0348(17)$ & $0.0488(18)$ & $0.0030(15)$ & $0.0095(18)$ & $-0.0110(18)$ \\
\hline C11 & $0.0329(18)$ & $0.0360(18)$ & $0.043(2)$ & $-0.0054(15)$ & $0.0019(13)$ & $-0.0144(14)$ \\
\hline $\mathrm{C} 12$ & $0.0243(16)$ & $0.0316(16)$ & $0.0399(18)$ & $-0.0013(13)$ & $0.0045(13)$ & $-0.0033(14)$ \\
\hline $\mathrm{C} 13$ & $0.0281(17)$ & $0.0462(18)$ & $0.0343(16)$ & $-0.0019(14)$ & $-0.0006(14)$ & $-0.0098(16)$ \\
\hline $\mathrm{C} 14$ & $0.0370(18)$ & $0.0350(18)$ & $0.0388(17)$ & $-0.0061(15)$ & $0.0014(14)$ & $0.0035(14)$ \\
\hline $\mathrm{C} 15$ & $0.0313(17)$ & $0.0331(18)$ & $0.0383(19)$ & $0.0017(14)$ & $0.0015(13)$ & $-0.0001(13)$ \\
\hline $\mathrm{C} 16$ & $0.0264(17)$ & $0.0401(19)$ & $0.039(2)$ & $-0.0046(14)$ & $0.0011(12)$ & $-0.0058(13)$ \\
\hline $\mathrm{C} 17$ & $0.0238(17)$ & $0.043(2)$ & $0.0422(17)$ & $0.0014(14)$ & $-0.0007(13)$ & $-0.0082(16)$ \\
\hline $\mathrm{C} 18$ & $0.0239(16)$ & $0.0350(18)$ & $0.0351(18)$ & 0.0007 (14) & $0.0046(12)$ & $-0.0021(13)$ \\
\hline C19 & $0.0251(16)$ & $0.0315(17)$ & $0.0362(18)$ & $-0.0001(14)$ & $0.0040(11)$ & $0.0005(13)$ \\
\hline
\end{tabular}

Geometric parameters $\left(\AA,{ }^{\circ}\right)$

\begin{tabular}{llll}
\hline $\mathrm{Co} 1-\mathrm{O} 1^{\mathrm{i}}$ & $2.037(2)$ & $\mathrm{C} 3-\mathrm{C} 8$ & $1.514(4)$ \\
$\mathrm{Co} 1-\mathrm{O} 2^{\mathrm{ii}}$ & $2.054(2)$ & $\mathrm{C} 3-\mathrm{H} 3 \mathrm{~A}$ & 0.9700 \\
$\mathrm{Co} 1-\mathrm{N} 3$ & $2.130(2)$ & $\mathrm{C} 3-\mathrm{H} 3 \mathrm{~B}$ & 0.9700 \\
$\mathrm{Co} 1-\mathrm{O} 4$ & $2.142(2)$ & $\mathrm{C} 4-\mathrm{C} 5$ & $1.374(4)$ \\
$\mathrm{Co} 1-\mathrm{N} 2^{\mathrm{iii}}$ & $2.158(3)$ & $\mathrm{C} 4-\mathrm{C} 6$ & $1.386(4)$
\end{tabular}




\begin{tabular}{|c|c|c|c|}
\hline $\mathrm{Co} 1-\mathrm{O} 5$ & $2.252(2)$ & $\mathrm{C} 4-\mathrm{H} 4 \mathrm{~A}$ & 0.9300 \\
\hline $\mathrm{N} 1-\mathrm{C} 12$ & $1.347(4)$ & $\mathrm{C} 5-\mathrm{H} 5 \mathrm{~A}$ & 0.9300 \\
\hline $\mathrm{N} 1-\mathrm{N} 2$ & $1.348(3)$ & $\mathrm{C} 6-\mathrm{C} 7$ & $1.384(4)$ \\
\hline $\mathrm{N} 1-\mathrm{H} 1 \mathrm{~A}$ & 0.8600 & $\mathrm{C} 7-\mathrm{H} 7 \mathrm{~A}$ & 0.9300 \\
\hline $\mathrm{N} 2-\mathrm{C} 10$ & $1.325(4)$ & $\mathrm{C} 9-\mathrm{C} 12$ & $1.374(4)$ \\
\hline $\mathrm{N} 2-\mathrm{Co}^{1 \mathrm{iv}}$ & $2.158(3)$ & $\mathrm{C} 9-\mathrm{C} 10$ & $1.391(4)$ \\
\hline N3- $\mathrm{C} 16$ & $1.333(4)$ & C9-H9A & 0.9300 \\
\hline $\mathrm{N} 3-\mathrm{C} 13$ & $1.338(4)$ & $\mathrm{C} 10-\mathrm{H} 10 \mathrm{~A}$ & 0.9300 \\
\hline $\mathrm{O} 1-\mathrm{C} 8$ & $1.251(3)$ & $\mathrm{C} 11-\mathrm{C} 19$ & $1.376(4)$ \\
\hline $\mathrm{O} 1-\mathrm{Co}^{\mathrm{v}}$ & $2.037(2)$ & $\mathrm{C} 11-\mathrm{C} 16$ & $1.381(4)$ \\
\hline $\mathrm{O} 2-\mathrm{C} 8$ & $1.253(4)$ & $\mathrm{C} 11-\mathrm{H} 11 \mathrm{~A}$ & 0.9300 \\
\hline $\mathrm{O} 2-\mathrm{Co}^{\mathrm{vi}}$ & $2.054(2)$ & $\mathrm{C} 12-\mathrm{C} 19$ & $1.467(4)$ \\
\hline $\mathrm{O} 3-\mathrm{C} 6$ & $1.374(3)$ & $\mathrm{C} 13-\mathrm{C} 17$ & $1.380(4)$ \\
\hline $\mathrm{O} 3-\mathrm{C} 3$ & $1.420(3)$ & $\mathrm{C} 13-\mathrm{H} 13 \mathrm{~A}$ & 0.9300 \\
\hline $\mathrm{O} 4-\mathrm{C} 18$ & $1.261(4)$ & $\mathrm{C} 14-\mathrm{C} 15$ & $1.316(4)$ \\
\hline $\mathrm{O} 5-\mathrm{C} 18$ & $1.266(3)$ & $\mathrm{C} 14-\mathrm{C} 18$ & $1.482(4)$ \\
\hline $\mathrm{C} 1-\mathrm{C} 5$ & $1.375(4)$ & $\mathrm{C} 14-\mathrm{H} 14 \mathrm{~A}$ & 0.9300 \\
\hline $\mathrm{C} 1-\mathrm{C} 2$ & $1.394(4)$ & C15-H15A & 0.9300 \\
\hline $\mathrm{C} 1-\mathrm{H} 1 \mathrm{~B}$ & 0.9300 & $\mathrm{C} 16-\mathrm{H} 16 \mathrm{~A}$ & 0.9300 \\
\hline $\mathrm{C} 2-\mathrm{C} 7$ & $1.400(4)$ & C17-C19 & $1.390(4)$ \\
\hline $\mathrm{C} 2-\mathrm{C} 15$ & $1.476(4)$ & C17-H17A & 0.9300 \\
\hline $\mathrm{O} 1^{\mathrm{i}}-\mathrm{Co} 1-\mathrm{O} 2^{\mathrm{ii}}$ & $102.24(8)$ & $\mathrm{C} 4-\mathrm{C} 5-\mathrm{H} 5 \mathrm{~A}$ & 119.5 \\
\hline $\mathrm{O} 1-\mathrm{Co} 1-\mathrm{N} 3$ & $91.33(9)$ & $\mathrm{C} 1-\mathrm{C} 5-\mathrm{H} 5 \mathrm{~A}$ & 119.5 \\
\hline $\mathrm{O} 2^{\mathrm{ii}}-\mathrm{Co} 1-\mathrm{N} 3$ & $92.82(9)$ & $\mathrm{O} 3-\mathrm{C} 6-\mathrm{C} 7$ & $125.7(3)$ \\
\hline $\mathrm{O} 1-\mathrm{Co} 1-\mathrm{O} 4$ & $95.01(8)$ & $\mathrm{O} 3-\mathrm{C} 6-\mathrm{C} 4$ & $114.6(3)$ \\
\hline $\mathrm{O} 2^{\mathrm{ii}}-\mathrm{Co} 1-\mathrm{O} 4$ & $162.75(8)$ & $\mathrm{C} 7-\mathrm{C} 6-\mathrm{C} 4$ & $119.7(3)$ \\
\hline $\mathrm{N} 3-\mathrm{Co} 1-\mathrm{O} 4$ & $87.08(9)$ & $\mathrm{C} 6-\mathrm{C} 7-\mathrm{C} 2$ & $120.1(3)$ \\
\hline $\mathrm{O} 1^{\mathrm{i}}-\mathrm{Co} 1-\mathrm{N} 2^{\mathrm{iii}}$ & $87.48(10)$ & $\mathrm{C} 6-\mathrm{C} 7-\mathrm{H} 7 \mathrm{~A}$ & 119.9 \\
\hline $\mathrm{O} 2^{\mathrm{ii}}-\mathrm{Co} 1-\mathrm{N} 2^{\mathrm{iii}}$ & $87.89(9)$ & $\mathrm{C} 2-\mathrm{C} 7-\mathrm{H} 7 \mathrm{~A}$ & 119.9 \\
\hline $\mathrm{N} 3-\mathrm{Co} 1-\mathrm{N} 2^{\mathrm{iii}}$ & $178.72(11)$ & $\mathrm{O} 1-\mathrm{C} 8-\mathrm{O} 2$ & $125.2(3)$ \\
\hline $\mathrm{O} 4-\mathrm{Co} 1-\mathrm{N} 2^{\mathrm{iii}}$ & $92.56(9)$ & $\mathrm{O} 1-\mathrm{C} 8-\mathrm{C} 3$ & $115.3(3)$ \\
\hline $\mathrm{O} 1{ }^{\mathrm{i}}-\mathrm{Co} 1-\mathrm{O} 5$ & $152.48(8)$ & $\mathrm{O} 2-\mathrm{C} 8-\mathrm{C} 3$ & $119.5(3)$ \\
\hline $\mathrm{O} 2^{\mathrm{ii}}-\mathrm{Co} 1-\mathrm{O} 5$ & $103.33(8)$ & $\mathrm{C} 12-\mathrm{C} 9-\mathrm{C} 10$ & $105.3(3)$ \\
\hline $\mathrm{N} 3-\mathrm{Co} 1-\mathrm{O} 5$ & $97.42(9)$ & $\mathrm{C} 12-\mathrm{C} 9-\mathrm{H} 9 \mathrm{~A}$ & 127.3 \\
\hline $\mathrm{O} 4-\mathrm{Co} 1-\mathrm{O} 5$ & $59.66(8)$ & $\mathrm{C} 10-\mathrm{C} 9-\mathrm{H} 9 \mathrm{~A}$ & 127.3 \\
\hline $\mathrm{N} 2^{\mathrm{iii}-\mathrm{Co} 1-\mathrm{O} 5}$ & $83.45(9)$ & $\mathrm{N} 2-\mathrm{C} 10-\mathrm{C} 9$ & $111.3(3)$ \\
\hline $\mathrm{C} 12-\mathrm{N} 1-\mathrm{N} 2$ & $112.2(2)$ & $\mathrm{N} 2-\mathrm{C} 10-\mathrm{H} 10 \mathrm{~A}$ & 124.4 \\
\hline $\mathrm{C} 12-\mathrm{N} 1-\mathrm{H} 1 \mathrm{~A}$ & 123.9 & $\mathrm{C} 9-\mathrm{C} 10-\mathrm{H} 10 \mathrm{~A}$ & 124.4 \\
\hline $\mathrm{N} 2-\mathrm{N} 1-\mathrm{H} 1 \mathrm{~A}$ & 123.9 & $\mathrm{C} 19-\mathrm{C} 11-\mathrm{C} 16$ & $120.1(3)$ \\
\hline $\mathrm{C} 10-\mathrm{N} 2-\mathrm{N} 1$ & $104.9(2)$ & $\mathrm{C} 19-\mathrm{C} 11-\mathrm{H} 11 \mathrm{~A}$ & 120.0 \\
\hline $\mathrm{C} 10-\mathrm{N} 2-\mathrm{Co}^{\mathrm{iv}}$ & $131.5(2)$ & $\mathrm{C} 16-\mathrm{C} 11-\mathrm{H} 11 \mathrm{~A}$ & 120.0 \\
\hline $\mathrm{N} 1-\mathrm{N} 2-\mathrm{Co}^{\mathrm{iv}}$ & $117.32(19)$ & $\mathrm{N} 1-\mathrm{C} 12-\mathrm{C} 9$ & $106.3(3)$ \\
\hline $\mathrm{C} 16-\mathrm{N} 3-\mathrm{C} 13$ & $117.4(3)$ & $\mathrm{N} 1-\mathrm{C} 12-\mathrm{C} 19$ & $122.1(3)$ \\
\hline $\mathrm{C} 16-\mathrm{N} 3-\mathrm{Co} 1$ & $121.0(2)$ & $\mathrm{C} 9-\mathrm{C} 12-\mathrm{C} 19$ & $131.1(3)$ \\
\hline $\mathrm{C} 13-\mathrm{N} 3-\mathrm{Co} 1$ & $121.4(2)$ & $\mathrm{N} 3-\mathrm{C} 13-\mathrm{C} 17$ & $123.1(3)$ \\
\hline $\mathrm{C} 8-\mathrm{O} 1-\mathrm{Co}^{\mathrm{v}}$ & $132.5(2)$ & $\mathrm{N} 3-\mathrm{C} 13-\mathrm{H} 13 \mathrm{~A}$ & 118.5 \\
\hline $\mathrm{C} 8-\mathrm{O} 2-\mathrm{Co}^{\mathrm{vi}}$ & $124.8(2)$ & $\mathrm{C} 17-\mathrm{C} 13-\mathrm{H} 13 \mathrm{~A}$ & 118.5 \\
\hline
\end{tabular}




\begin{tabular}{|c|c|c|c|}
\hline $\mathrm{C} 6-\mathrm{O} 3-\mathrm{C} 3$ & $117.6(2)$ & $\mathrm{C} 15-\mathrm{C} 14-\mathrm{C} 18$ & $125.6(3)$ \\
\hline $\mathrm{C} 18-\mathrm{O} 4-\mathrm{Co} 1$ & $92.70(18)$ & $\mathrm{C} 15-\mathrm{C} 14-\mathrm{H} 14 \mathrm{~A}$ & 117.2 \\
\hline $\mathrm{C} 18-\mathrm{O} 5-\mathrm{Co} 1$ & $87.59(18)$ & $\mathrm{C} 18-\mathrm{C} 14-\mathrm{H} 14 \mathrm{~A}$ & 117.2 \\
\hline $\mathrm{C} 5-\mathrm{C} 1-\mathrm{C} 2$ & $119.9(3)$ & $\mathrm{C} 14-\mathrm{C} 15-\mathrm{C} 2$ & $125.4(3)$ \\
\hline $\mathrm{C} 5-\mathrm{C} 1-\mathrm{H} 1 \mathrm{~B}$ & 120.1 & $\mathrm{C} 14-\mathrm{C} 15-\mathrm{H} 15 \mathrm{~A}$ & 117.3 \\
\hline $\mathrm{C} 2-\mathrm{C} 1-\mathrm{H} 1 \mathrm{~B}$ & 120.1 & $\mathrm{C} 2-\mathrm{C} 15-\mathrm{H} 15 \mathrm{~A}$ & 117.3 \\
\hline $\mathrm{C} 1-\mathrm{C} 2-\mathrm{C} 7$ & $119.2(3)$ & N3-C16-C11 & $122.8(3)$ \\
\hline $\mathrm{C} 1-\mathrm{C} 2-\mathrm{C} 15$ & $121.5(3)$ & $\mathrm{N} 3-\mathrm{C} 16-\mathrm{H} 16 \mathrm{~A}$ & 118.6 \\
\hline $\mathrm{C} 7-\mathrm{C} 2-\mathrm{C} 15$ & $119.3(3)$ & $\mathrm{C} 11-\mathrm{C} 16-\mathrm{H} 16 \mathrm{~A}$ & 118.6 \\
\hline $\mathrm{O} 3-\mathrm{C} 3-\mathrm{C} 8$ & $115.4(3)$ & $\mathrm{C} 13-\mathrm{C} 17-\mathrm{C} 19$ & $119.4(3)$ \\
\hline $\mathrm{O} 3-\mathrm{C} 3-\mathrm{H} 3 \mathrm{~A}$ & 108.4 & $\mathrm{C} 13-\mathrm{C} 17-\mathrm{H} 17 \mathrm{~A}$ & 120.3 \\
\hline $\mathrm{C} 8-\mathrm{C} 3-\mathrm{H} 3 \mathrm{~A}$ & 108.4 & $\mathrm{C} 19-\mathrm{C} 17-\mathrm{H} 17 \mathrm{~A}$ & 120.3 \\
\hline $\mathrm{O} 3-\mathrm{C} 3-\mathrm{H} 3 \mathrm{~B}$ & 108.4 & $\mathrm{O} 4-\mathrm{C} 18-\mathrm{O} 5$ & $120.0(3)$ \\
\hline $\mathrm{C} 8-\mathrm{C} 3-\mathrm{H} 3 \mathrm{~B}$ & 108.4 & $\mathrm{O} 4-\mathrm{C} 18-\mathrm{C} 14$ & $118.3(3)$ \\
\hline $\mathrm{H} 3 \mathrm{~A}-\mathrm{C} 3-\mathrm{H} 3 \mathrm{~B}$ & 107.5 & $\mathrm{O} 5-\mathrm{C} 18-\mathrm{C} 14$ & $121.7(3)$ \\
\hline $\mathrm{C} 5-\mathrm{C} 4-\mathrm{C} 6$ & $120.1(3)$ & $\mathrm{C} 11-\mathrm{C} 19-\mathrm{C} 17$ & $117.1(3)$ \\
\hline $\mathrm{C} 5-\mathrm{C} 4-\mathrm{H} 4 \mathrm{~A}$ & 120.0 & $\mathrm{C} 11-\mathrm{C} 19-\mathrm{C} 12$ & $120.6(3)$ \\
\hline $\mathrm{C} 6-\mathrm{C} 4-\mathrm{H} 4 \mathrm{~A}$ & 120.0 & $\mathrm{C} 17-\mathrm{C} 19-\mathrm{C} 12$ & $122.0(3)$ \\
\hline $\mathrm{C} 4-\mathrm{C} 5-\mathrm{C} 1$ & $120.9(3)$ & & \\
\hline $\mathrm{C} 12-\mathrm{N} 1-\mathrm{N} 2-\mathrm{C} 10$ & $1.1(3)$ & $\mathrm{C} 10-\mathrm{C} 9-\mathrm{C} 12-\mathrm{N} 1$ & $0.5(4)$ \\
\hline $\mathrm{C} 12-\mathrm{N} 1-\mathrm{N} 2-\mathrm{Co}^{\mathrm{iv}}$ & $-154.4(2)$ & $\mathrm{C} 10-\mathrm{C} 9-\mathrm{C} 12-\mathrm{C} 19$ & $-170.5(3)$ \\
\hline $\mathrm{C} 5-\mathrm{C} 1-\mathrm{C} 2-\mathrm{C} 7$ & $-1.3(5)$ & $\mathrm{C} 16-\mathrm{N} 3-\mathrm{C} 13-\mathrm{C} 17$ & $-2.1(5)$ \\
\hline $\mathrm{C} 5-\mathrm{C} 1-\mathrm{C} 2-\mathrm{C} 15$ & $179.6(3)$ & $\mathrm{Co} 1-\mathrm{N} 3-\mathrm{C} 13-\mathrm{C} 17$ & $172.6(3)$ \\
\hline $\mathrm{C} 6-\mathrm{O} 3-\mathrm{C} 3-\mathrm{C} 8$ & $73.1(3)$ & $\mathrm{C} 18-\mathrm{C} 14-\mathrm{C} 15-\mathrm{C} 2$ & $-179.0(3)$ \\
\hline $\mathrm{C} 6-\mathrm{C} 4-\mathrm{C} 5-\mathrm{C} 1$ & $1.2(5)$ & $\mathrm{C} 1-\mathrm{C} 2-\mathrm{C} 15-\mathrm{C} 14$ & $21.2(5)$ \\
\hline $\mathrm{C} 2-\mathrm{C} 1-\mathrm{C} 5-\mathrm{C} 4$ & $0.9(5)$ & $\mathrm{C} 7-\mathrm{C} 2-\mathrm{C} 15-\mathrm{C} 14$ & $-157.9(3)$ \\
\hline $\mathrm{C} 3-\mathrm{O} 3-\mathrm{C} 6-\mathrm{C} 7$ & $-3.8(4)$ & $\mathrm{C} 13-\mathrm{N} 3-\mathrm{C} 16-\mathrm{C} 11$ & $1.1(4)$ \\
\hline $\mathrm{C} 3-\mathrm{O} 3-\mathrm{C} 6-\mathrm{C} 4$ & $176.1(3)$ & $\mathrm{Co} 1-\mathrm{N} 3-\mathrm{C} 16-\mathrm{C} 11$ & $-173.7(2)$ \\
\hline $\mathrm{C} 5-\mathrm{C} 4-\mathrm{C} 6-\mathrm{O} 3$ & $177.0(3)$ & $\mathrm{C} 19-\mathrm{C} 11-\mathrm{C} 16-\mathrm{N} 3$ & $2.0(5)$ \\
\hline $\mathrm{C} 5-\mathrm{C} 4-\mathrm{C} 6-\mathrm{C} 7$ & $-3.1(5)$ & $\mathrm{N} 3-\mathrm{C} 13-\mathrm{C} 17-\mathrm{C} 19$ & $0.0(5)$ \\
\hline $\mathrm{O} 3-\mathrm{C} 6-\mathrm{C} 7-\mathrm{C} 2$ & $-177.4(3)$ & $\mathrm{Co} 1-\mathrm{O} 4-\mathrm{C} 18-\mathrm{O} 5$ & $3.0(3)$ \\
\hline $\mathrm{C} 4-\mathrm{C} 6-\mathrm{C} 7-\mathrm{C} 2$ & $2.7(4)$ & $\mathrm{Co} 1-\mathrm{O} 4-\mathrm{C} 18-\mathrm{C} 14$ & $-178.0(2)$ \\
\hline $\mathrm{C} 1-\mathrm{C} 2-\mathrm{C} 7-\mathrm{C} 6$ & $-0.5(4)$ & $\mathrm{Co} 1-\mathrm{O} 5-\mathrm{C} 18-\mathrm{O} 4$ & $-2.8(3)$ \\
\hline $\mathrm{C} 15-\mathrm{C} 2-\mathrm{C} 7-\mathrm{C} 6$ & $178.6(3)$ & $\mathrm{Co} 1-\mathrm{O} 5-\mathrm{C} 18-\mathrm{C} 14$ & $178.2(3)$ \\
\hline $\mathrm{Co}^{\mathrm{v}}-\mathrm{O} 1-\mathrm{C} 8-\mathrm{O} 2$ & $124.6(3)$ & $\mathrm{C} 15-\mathrm{C} 14-\mathrm{C} 18-\mathrm{O} 4$ & $178.6(3)$ \\
\hline $\mathrm{Co}^{\mathrm{v}}-\mathrm{O} 1-\mathrm{C} 8-\mathrm{C} 3$ & $-55.7(3)$ & $\mathrm{C} 15-\mathrm{C} 14-\mathrm{C} 18-\mathrm{O} 5$ & $-2.4(5)$ \\
\hline $\mathrm{Co}^{\mathrm{vi}}-\mathrm{O} 2-\mathrm{C} 8-\mathrm{O} 1$ & $5.7(4)$ & $\mathrm{C} 16-\mathrm{C} 11-\mathrm{C} 19-\mathrm{C} 17$ & $-4.0(5)$ \\
\hline $\mathrm{Co} 1^{\mathrm{vi}}-\mathrm{O} 2-\mathrm{C} 8-\mathrm{C} 3$ & $-174.00(19)$ & $\mathrm{C} 16-\mathrm{C} 11-\mathrm{C} 19-\mathrm{C} 12$ & $170.6(3)$ \\
\hline $\mathrm{O} 3-\mathrm{C} 3-\mathrm{C} 8-\mathrm{O} 1$ & $-168.3(2)$ & $\mathrm{C} 13-\mathrm{C} 17-\mathrm{C} 19-\mathrm{C} 11$ & $3.0(5)$ \\
\hline $\mathrm{O} 3-\mathrm{C} 3-\mathrm{C} 8-\mathrm{O} 2$ & $11.4(4)$ & $\mathrm{C} 13-\mathrm{C} 17-\mathrm{C} 19-\mathrm{C} 12$ & $-171.4(3)$ \\
\hline $\mathrm{N} 1-\mathrm{N} 2-\mathrm{C} 10-\mathrm{C} 9$ & $-0.7(4)$ & $\mathrm{N} 1-\mathrm{C} 12-\mathrm{C} 19-\mathrm{C} 11$ & $-156.1(3)$ \\
\hline $\mathrm{Co}^{\mathrm{iv}}-\mathrm{N} 2-\mathrm{C} 10-\mathrm{C} 9$ & $149.7(3)$ & $\mathrm{C} 9-\mathrm{C} 12-\mathrm{C} 19-\mathrm{C} 11$ & $13.7(5)$ \\
\hline $\mathrm{C} 12-\mathrm{C} 9-\mathrm{C} 10-\mathrm{N} 2$ & $0.2(4)$ & $\mathrm{N} 1-\mathrm{C} 12-\mathrm{C} 19-\mathrm{C} 17$ & $18.2(5)$ \\
\hline
\end{tabular}




\section{supporting information}

$\begin{array}{llll}\mathrm{N} 2-\mathrm{N} 1-\mathrm{C} 12-\mathrm{C} 9 & -1.0(4) & \mathrm{C} 9-\mathrm{C} 12-\mathrm{C} 19-\mathrm{C} 17 & -172.0(3) \\ \mathrm{N} 2-\mathrm{N} 1-\mathrm{C} 12-\mathrm{C} 19 & 171.0(3) & & \end{array}$

Symmetry codes: (i) $x-1 / 4,-y+1 / 4, z-5 / 4$; (ii) $x-1 / 4,-y+1 / 4, z-1 / 4$; (iii) $x+1 / 4,-y+1 / 4, z-3 / 4$; (iv) $x-1 / 4,-y+1 / 4, z+3 / 4$; (v) $x+1 / 4,-y+1 / 4, z+5 / 4$; (vi) $x+1 / 4,-y+1 / 4, z+1 / 4$.

Hydrogen-bond geometry $\left(\AA,{ }^{\circ}\right)$

\begin{tabular}{lllll}
\hline$D-\mathrm{H} \cdots A$ & $D-\mathrm{H}$ & $\mathrm{H} \cdots A$ & $D \cdots A$ & $D-\mathrm{H} \cdots A$ \\
\hline $\mathrm{N} 1-\mathrm{H} 1 A \cdots \mathrm{O} 5^{\mathrm{ii}}$ & 0.86 & 2.05 & $2.869(3)$ & 159 \\
\hline
\end{tabular}

Symmetry code: (ii) $x-1 / 4,-y+1 / 4, z-1 / 4$. 